Vol.01/ No. 01

Pages: $30-40$

https://www.irojournals.com/iroeea

DOI: https://doi.org/10.36548/jeea.2019.1.004

\title{
ADAPTABLE MULTI-TOUCH TECHNOLOGY SYSTEM FOR TOUCH SENSITIVE DEVICES
}

\author{
Mr. R. Vinothkanna, \\ Department of ECE, Vivekanandha College of Technology for Women, \\ Namakal, India. \\ Email: rvinothkannaphd@gmail.com
}

\begin{abstract}
The touch sensing technology that has paved way for a user friendly interaction with the components has become a more prominent option in the recent days due to its capability of providing a direct communication by just one touch and its compatibility with the users of all age and different level of skills. The touch technology can be single or multi and supported by variety of technologies, such as resistive, capacitive, acoustic and infrared. Among all the technologies the capacitive sensor are widely opted as they possess a low hysteresis, good repeatability and faster response time. The paper also puts forward adaptable multi-touch technology system for the touch sensitive devices, by proposing a flexible capacitive touch sensor to make touch sensitive devices more users friendly with the a highest degree of sensitivity to the touch and satisfactory performance for a broader range of applications with the heightened durability.
\end{abstract}

Keywords: Touch Sensitive Devices, Single Touch Technology, Multi- Touch Technology, Capacitive Sensors, Nano Fibers, PVdf-PTrFE

\section{INTRODUCTION}

In case of electronic on making decision to shift from an older interface to a newer one either it is for a personal use or the commercial purpose the equipment's with the touch sensitiveness have become more prominent options. As the touch sensitive equipment's are more compatible with the users of all ages with different level of skills. The touch sensitive component ensures the direct accessibility to the programs by just touching the surface providing an enhanced direct and the natural communication that is simple and spontaneous. The touch sensitive devices usually hold a surface that is the touch screen. The touch screen is nothing but an electronic visual display with the capability of identifying and positioning the touch over the surface. The Pickering, J in [1] his paper details that the human usual activity of pointing for the communication serves as the starting point for the development of the touch sensitive devices, he also notifies that the touch sensitive devices ensures the electronic devices to sense and utilize the 'natural pointing instincts' of communication precisely. Caporusso et al quotes that even the "deaf and blind individuals initially use the touch to extend communication and interact with the world. The signs of touch would be 
Journal of Electrical Engineering and Automation (EEA) (2019)

Vol.01/ No. 01

Pages: $30-40$

https://www.irojournals.com/iroeea

DOI: https://doi.org/10.36548/jeea.2019.1.004

very much useful in the basic communication". The fig. 1 below shows the components and the operation of the touch sensitive devices.

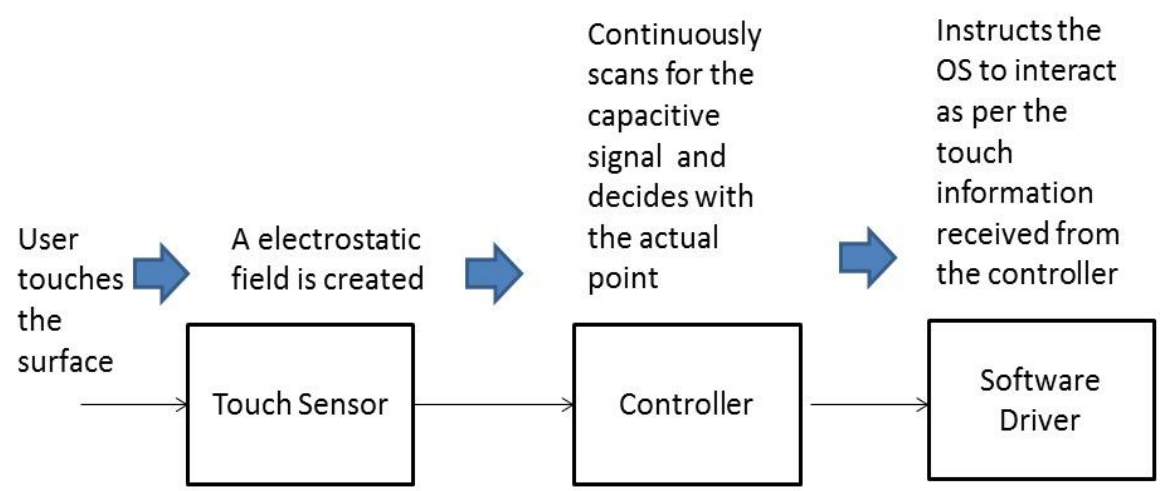

Fig .1 Components and the Operation of Touch Sensitive Devices

Though the touch sensitive device has emerged long back, still proceeds come up with new capabilities by including latest advancements in them.

So the paper puts forward the adaptable multi-touch technology system for the touch sensitive devices, by proposing a flexible capacitive touch sensor to make touch sensitive devices more user friendly, overcoming the default defects in the capacitive touch sensors.

The rest of the paper below is arranged with the 2. Providing the related works that describes the details of the touch sensitive devices and the variety of technologies used 3.Providing the proposed work, 4. The results, 5. Provides Conclusion describing the summary if the work done

\section{RELATED WORKS}

Pickering, J. et al [1] The author elaborates the review on the capability of the touch sensing in the displays and the various styles of their uses in satisfying the needs of the application and their designing Schaeffer et al [2] the author develops a sensor integrated robot arm to recognize the need of the elderly and the disabled 
Journal of Electrical Engineering and Automation (EEA) (2019)

Vol.01/ No. 01

Pages: $30-40$

https://www.irojournals.com/iroeea

DOI: https://doi.org/10.36548/jeea.2019.1.004

Caporusso, et al [3] the author has put forward a system integrated with the already existing touch and the gesture based languages to bring out a unique device that supports the diverse societies with an reasonable clarifications. To have a more elaborate view of the touch sensitive devices let us move into the descriptions surveyed from the literature to have a basic knowledge of the touch sensitive devices, the technologies used in them and the categories of the

\subsection{TOUCH SENSITIVE DEVICES}

Often recognized as the rectangular device that is flat in shape and reciprocating immediately to each touch that is done by the naked finger or using any other tools. Once the surface experiences a touch it immediately transmits the point where the touch was felt or the co-ordinates where the touch was felt to the computer or the any other devices that are associated with the touch screen [4]. Some examples to add information about the touch sensitive devices are (i) lap top in which the touch device present in it is the touch pad and the movement of the finger across the pad would cause the cursor to move, (ii) mobile phones - that recognize the touch to enable the actions requested, (iii) smart machines such as the washing machines, vacuum cleaners, medical equipment's, industrial components, electronic voting machines, electronic skin, bio interactive technologies etc. that are touch sensitive and operate based on the operations that are touched etc. Multitude of examples could be added to the touch sensitive device, as the world today strives towards the modernization and easy access. The touch sensitive devices that behave as both the input and the output accessory are usually present as the top most layers on the display of the electronic equipment, the user can provide with the input instruction or a control instruction through a single or a multi touch so the touch sensitive device can be categorized into two types as the single touch sensitive devices and the multitouch sensitive devices. The fig. 2 below shows the touch sensitive model.

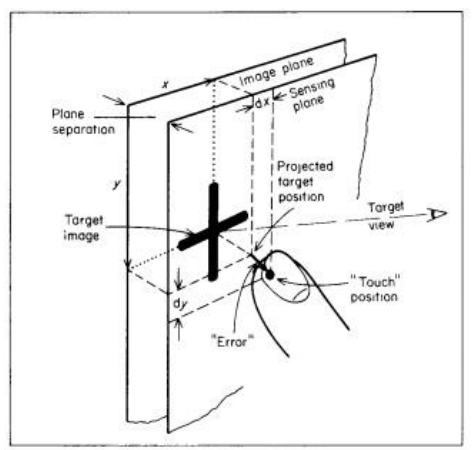

ISSN: 2582-3051 
Journal of Electrical Engineering and Automation (EEA) (2019)

Vol.01/ No. 01

Pages: $30-40$

https://www.irojournals.com/iroeea

DOI: https://doi.org/10.36548/jeea.2019.1.004

Fig .2 Touch sensitive Model [1]

The technologies used in the touch sensitive devices can be categorized into four types based on the sensors as the capacitive, restive, infrared and acoustic Schulz; et al [6] in his paper elaborates the different technologies available and how they are composed of, he explains the resistive touch screen is made up with the adjustable top layer and a firm lowest layer made with the ploythene and glass respectively. Further the construction of the capacitive screen is layered with the substance that reserves the electrical charges and contained with sensors at each intersection point of the row and the column. Where the other two technologies infrared and the acoustic is fitted with the IR LEDS, photo detectors and the transducers respectively, among all the technologies the capacitive sensor mostly preferred due to their low hysteresis, the faster response time and an enhanced repeatability. So Kim, et al [5] proposes the capacitive sensor that is tactile along with the adjusting capability designed for the applications with the multi-touch screen and Lewis et al [7] the author elaborates the uses of the touch screen technologies for the chemical module implementation in the high school as a motivation to the students. Savage, et al [8] put forward a "Midas fabricating custom capacitive touch sensors to prototype interactive objects." Harris et al [9] the author explains the capability of the multi-touch over the single touch in the by experimenting both among a group of people and projects the multi-touch to be superior to the single touch, as the multi-touch provides a simultaneous interaction with the digital world. Rimon et al [10] the author proffers a design of an input device that contains a touch sensitive sensor that flexibly operates on the single and the multi-touch detection with the single touch detected on the first period and the multi-touch detected on the second period of the sampling. Cataldi, et al [11] the study explaining the capacitive touch sensor that is reliable with the elongating the parallel plate is presented in the paper Sell, et al [12] the author presents a "Printed embedded transducers that is integrated with the capacitive touch sensors into the organic coating of metallic substrates" Abhishek et al [13] the author elaborates the usage of the capacitive touch sensor for the "glove based hand gesture recognition sign language translator". Jiang et al [14] the paper presents the "aligned PVdf-PTrFE nanofibers with the carbon nanotubes for an improved sensing in the piezoelectric directional strain sensing". Yang et al [15] carbon nanotube based bio sensors are developed in the paper, to have a faster response time The capacitive sensor that are more compatible and user friendly, to further enhance the sensitivity of the capacitive sensors the proposed method utilizes the PVdf-PTrFE electro spun nano fiber with the capacitive touch sensor, to improve its sensitivity causing adaptability in the multi touch technology.

\section{CAPACITIVE TOUCH SENSORS WITH PVDF-TRFE NANO FIBERS WITH THE CARBON NANOTUBES}

The Electrospun polyvinylidene fluoride-polytrifluoroethylene nanofiber membrane [14] with the carbon nanotube 
Journal of Electrical Engineering and Automation (EEA) (2019)

Vol.01/ No. 01

Pages: $30-40$

https://www.irojournals.com/iroeea

DOI: https://doi.org/10.36548/jeea.2019.1.004

[15] for the capacitive touch sensor is proposed in the paper to improve the touch sensitivity of the multi-touch technology and to improve its response time in order to make it more adaptable. To achieve this carbon nanotubes with the weight of .2 wt. \% were combined with the solution of the solution of the polyvinylidene fluoridepolytrifluoroethylene that was composed by the combining the polyvinylidene fluoride-polytrifluoroethylene dimethylformamide/acetone in the ratio of $3: 2$ with the weight fraction of the $21 \mathrm{wt} \%$. The weight of the carbon nanotube was taken much care as to note down that the carbon nanotube disperses evenly in the solution. The solvents after several hours of stirring and dispersing are layered over the nanofiber using the electro spinning method.

This is used as the dielectric medium in the capacitive touch sensor to improve it sensitivity and the performance when engaged with the multi-touch technology. The electrospun nanofiber membrane is coated on the top and the bottom with the ITO-PET to have record of the variations that occurs in the capacitance due to the external touch. This makes the capacitive touch sensor more flexible and elastic and compatible for numerous device applications that are flexible.

The permittivity of the electrospun nanofiber membrane that is composed using the PVdf-PTrFE and the carbon nanotube is given as shown in the equation (1) below.

$$
P_{c}=\frac{P_{p}}{\left|a-a_{p}\right| m}
$$

Where the $P_{p}$ is the permittivity of the PVdf-PTrFE and the $P_{c}$ is the permittivity of the electrospun nanofiber with the PVdf-PTrFE and the carbon nanotube. The equation (2) is framed in this regard is show below.

$$
P=P_{c}\left[1+\frac{V_{q}\left(P_{a}-P_{c}\right)}{P_{c}+\left(P_{a}-P_{c}\right)\left(1-V_{q}\right)}\right]
$$

Where $P$ gives the permittivity of the electrospun nanofiber framed without any external touch and $V_{q}$ gives the volume of the pores. The equation (3) gives the gives the relationship between the external touch and the sensor capacitance $\left(S_{c}\right)$. 
Journal of Electrical Engineering and Automation (EEA) (2019)

Vol.01/ No. 01

Pages: $30-40$

https://www.irojournals.com/iroeea

DOI: https://doi.org/10.36548/jeea.2019.1.004

$$
S_{c}=P_{a} P_{c} \frac{Q R}{R d-\sigma}\left[1+\frac{n\left(R d V_{q}-R d m^{2}-\sigma m^{2}\right)\left(P_{a}-P_{c}\right)}{R d n P_{c}+\left(P_{a}-P_{c}\right)\left(R d-R d V_{q}-R d m^{2}-\sigma m^{2}\right.}\right]
$$

Where the ' $R$ ' denotes the young's modulus, ' $d$ ' the distance between the electrodes in the top and the bottom layer, ' $\mathrm{Q}$ ' is the intermediate area between the electrode and the di-electric material, and ' $\mathrm{m}$ ' gives the Poisson ratio. The capacitive touch sensors capacitance is given by the equation (4) as shown below.

$$
c=\frac{P Q}{d}
$$

This ensures that the improvement in the sensitivity of the capacitive touch sensor by just enhancing the elasticity and by slightly altering the shape of the electrospun nanofiber that is framed by combining the PVdf-PTrFE and the carbon nanotubes. It is also possible to adjust the sensitivity of the touch sensors by integrating the dielectric area with the various kinds of porosity and the carbon nanotubes.

Further analysis of the electrospun nano fibers under the Atomic force microscope shows that the rough ness of the nano fiber increased or decreased by just increasing and decreasing the carbon nanotube additions and the increase in the permittivity is achieved by just maintaining the carbon nanotube concentration below the percolation threshold. The fig. 3 below shows the capacitive touch sensor using the electrospun nanofiber. 
Journal of Electrical Engineering and Automation (EEA) (2019)

Vol.01/ No. 01

Pages: $30-40$

https://www.irojournals.com/iroeea

DOI: https://doi.org/10.36548/jeea.2019.1.004

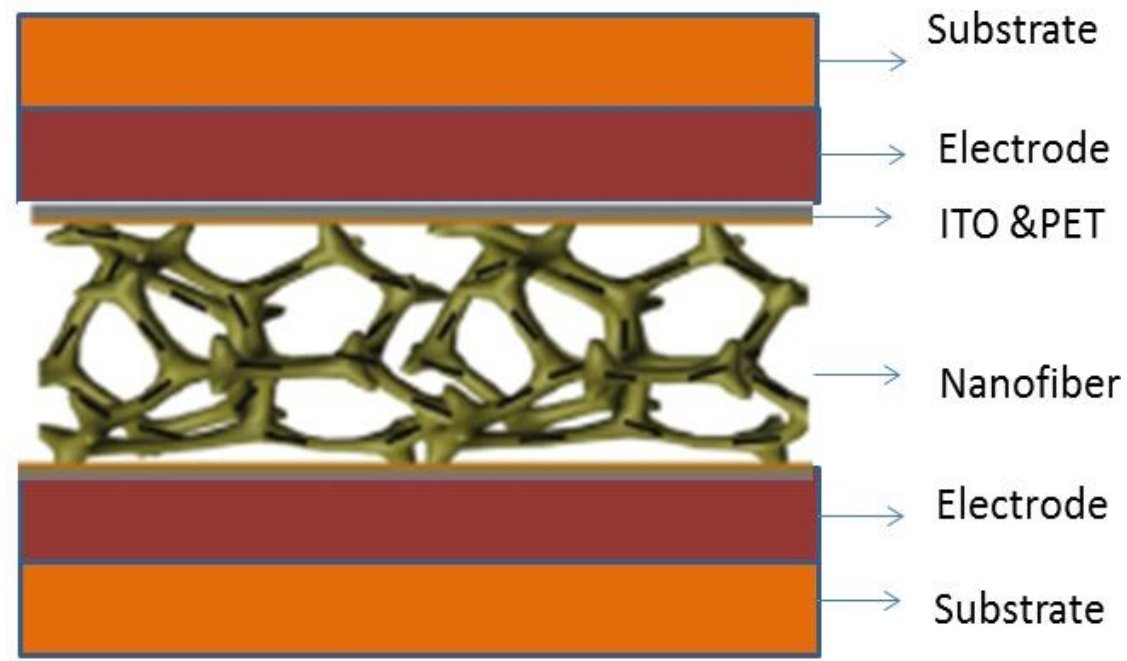

Fig .3 Capacitive Touch Sensors With electrospun Nanofiber

\section{RESULTS}

The capacitive touch sensor fabricated with the electrospun nano fiber is experimented to note down its sensitivity, with the initial energy of 100 joules at a frequency of $250 \mathrm{~Hz}$. Whenever a touch is felt it exerts pressure on the sensors and this causes a capacitance and that is noted down using the impedance analyzer. Each time an external touch is felt a pressure is exerted on the sensor that causes the capacitance to flow. The controller in the sensor units resolves the capacitive signals that flow and decides with accurate point that is touched. The proposed capacitive touch sensor integrated with the electrospun nanofiber composed of the PVdf-PTrFE and the carbon nanotubes provides an heightened sensitivity and a quick response by enhancing the permittivity and diminishing the ' $R$ ' of the PVdf-PTrFE and the carbon nanotube dielectric layer. The figure. 4 below shows the percentage of the sensitivity achieved under the different capacitance flow that is caused. 
Journal of Electrical Engineering and Automation (EEA) (2019)

Vol.01/ No. 01

Pages: $30-40$

https://www.irojournals.com/iroeea

DOI: https://doi.org/10.36548/jeea.2019.1.004

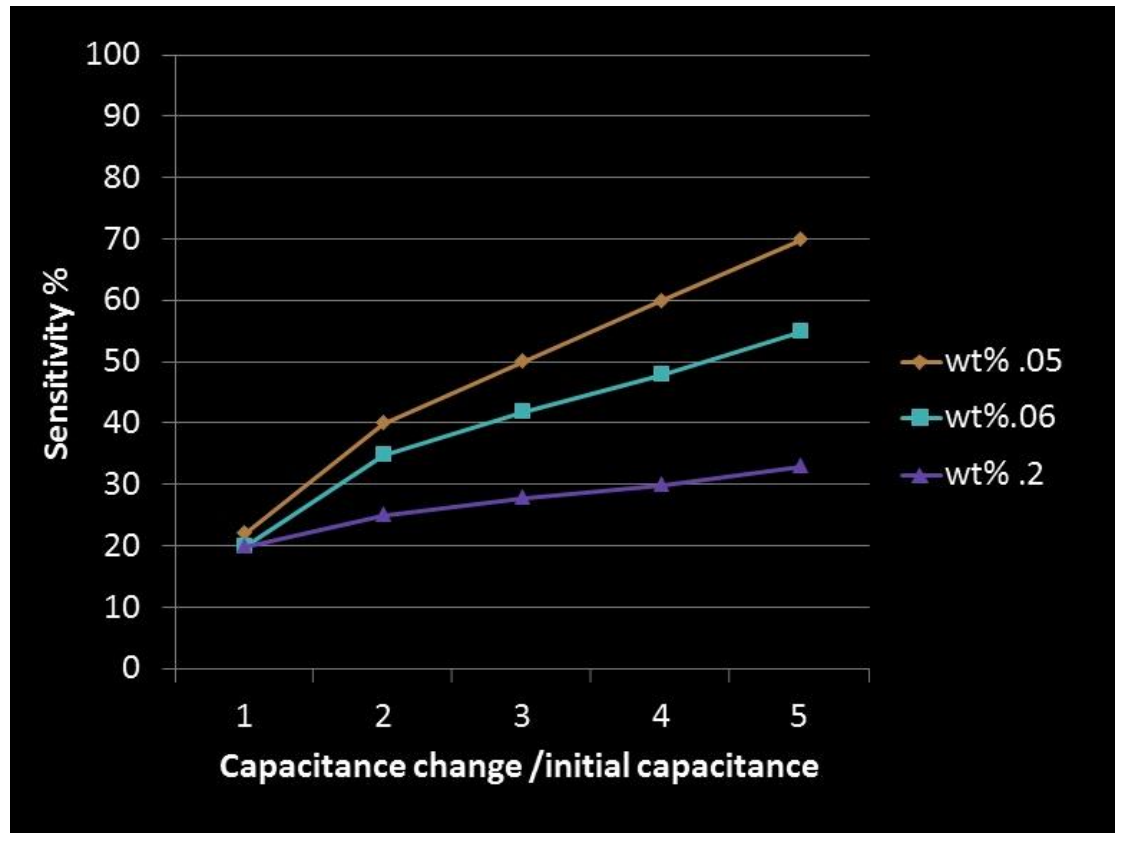

Fig .4 Percentage of Sensitivity

The fig .4 four shows the sensitivity percentage of the proposed capacitive touch sensor for different weight composition of the PVdf-PTrFE and the carbon nanotubes and the capacitance change observed. The sensitivity of the capacitive touch sensor is given in equation (5) that is shown below.

$$
\text { Sensitvity }=\frac{\delta\left(\frac{\Delta \text { capacitance }}{\text { intial capacitance }}\right)}{\delta(\text { pressure by external touch })}
$$


Journal of Electrical Engineering and Automation (EEA) (2019)

Vol.01/ No. 01

Pages: $30-40$

https://www.irojournals.com/iroeea

DOI: https://doi.org/10.36548/jeea.2019.1.004

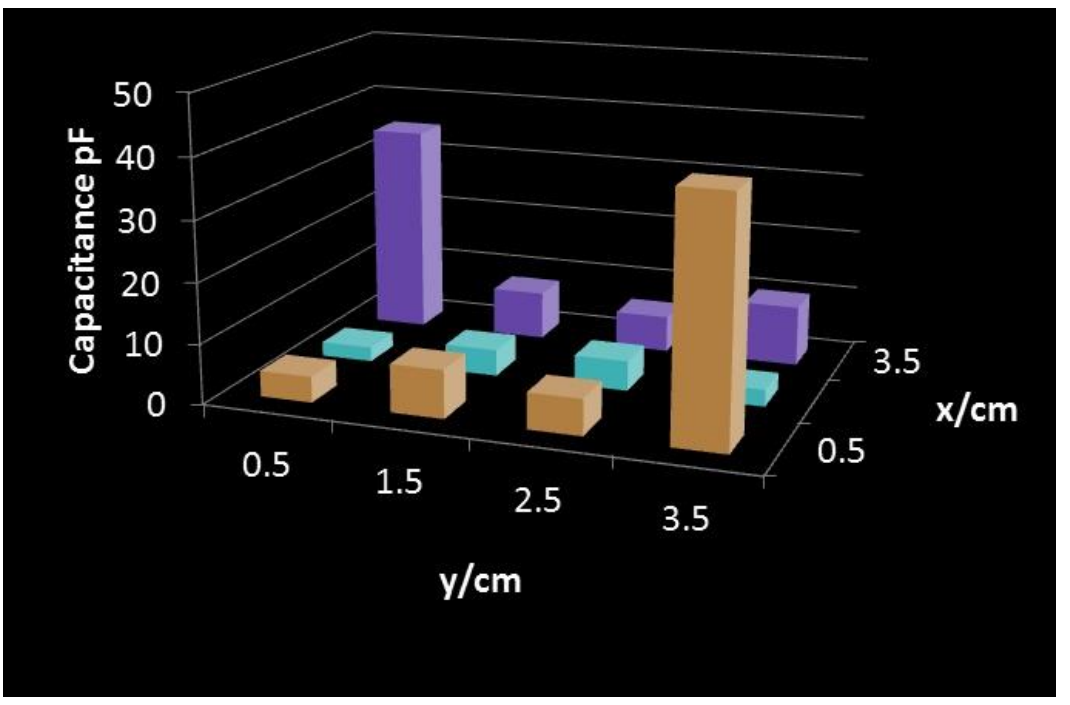

Fig .5 Capacitance Measured on Multi-touch Experienced on the Sensors

The fig.5 above shows the capacitance measured when the pressure is exerted on the sensors due to the multi-touch. The table. 1 below shows the sensitivity achieved and the response time of the proposed capacitive touch sensor with the PVdf-PTrFE carbon nanotubes and the other methods.

\begin{tabular}{|l|l|l|l|l|l|l|}
\hline Dielectric layer & AIR & PDMS & CNT/PDMS & $\begin{array}{l}\text { PYRAMID } \\
\text { PDMS }\end{array}$ & HYDROGEL & $\begin{array}{l}\text { ELCTROSPUN } \\
\text { NANOFIBER } \\
\text { (PVDF-TRFE } \\
\text { and CNT) }\end{array}$ \\
\hline Sensitivity \% & $30 \%$ & $25 \%$ & $15 \%$ & $55 \%$ & $35 \%$ & $80 \%$ \\
\hline $\begin{array}{l}\text { Response time in } \\
(\mathrm{ms})\end{array}$ & 55 & 64 & 60 & 50 & 40 & 25 \\
\hline
\end{tabular}

Table.1 Comparison of Sensitivity and Response Time

The table. 1 above makes clear that the proposed capacitive touch sensor with the PVdf-PTrFE carbon nanotubes achieves a heightened sensitivity and a faster response compared to the other materials used as the dielectric medium 
Journal of Electrical Engineering and Automation (EEA) (2019)

Vol.01/ No. 01

Pages: $30-40$

https://www.irojournals.com/iroeea

DOI: https://doi.org/10.36548/jeea.2019.1.004

instead of the PVdf-PTrFE carbon nanotubes.

This makes the capacitive touch sensor more compatible and adaptable for the multi-touch technology in applications such as the electronic skin, bio interactive technologies and soft robotics thus making the multi-touch technology adaptable.

\section{CONCLUSION}

The paper has proposed a highly sensitive and responsive capacitive touch sensor by integrating the electrospun nano fiber that is composed of the polyvinylidene fluoride-polytrifluoroethylene and the carbon nanotube to make it adaptable and compatible for the multi-touch technology. The flexibility of the nano membranes make the capacitive touch sensor more suitable and adaptable to the applications like the soft robotics, electronic skin and other applications that are related to the bio-interactive technologies. The further analysis of the sensor subjecting to the atomic force microscope and measuring the capacitance using the impedance analyzer, when exposed to an external touch shows that the proposed capacitive touch sensors with the polyvinylidene fluoride-polytrifluoroethylene and the carbon nanotube dielectric layer provides a heightened sensitivity and quicker response than the other types of dielectric layer that is silicon based.

\section{References}

[1] Pickering, J. A. "Touch-sensitive screens: the technologies and their application." International Journal of Man-Machine Studies 25, no. 3 (1986): 249-269.

[2] Schaeffer, C., and T. May. "Care-o-bot-a system for assisting elderly or disabled persons in home environments." Assistive technology on the threshold of the new millenium (1999).

[3] Caporusso, Nicholas, Luigi Biasi, Giovanni Cinquepalmi, Gianpaolo Francesco Trotta, Antonio Brunetti, and Vitoantonio Bevilacqua. "A wearable device supporting multiple touch-and gesture-based languages for the deaf-blind." In International Conference on Applied Human Factors and Ergonomics, pp. 32-41. Springer, Cham, 2017.

[4] https://www.encyclopedia.com/computing/dictionaries-thesauruses-pictures-and-press-releases/touchsensitive-device

[5] Kim, Hong-Ki, Seunggun Lee, and Kwang-Seok Yun. "Capacitive tactile sensor array for touch screen application." Sensors and Actuators A: Physical 165, no. 1 (2011): 2-7. 
Journal of Electrical Engineering and Automation (EEA) (2019)

Vol.01/ No. 01

Pages: $30-40$

https://www.irojournals.com/iroeea

DOI: https://doi.org/10.36548/jeea.2019.1.004

[6] Schulz, Stephen C., Anthony F. Chernefsky, and Bernard Geaghan. "Flexible capacitive touch sensor." U.S. Patent 6,819,316, issued November 16, 2004.

[7] Lewis, Maurica S., Jinhui Zhao, and Jin Kim Montclare. "Development and implementation of high school chemistry modules using touch-screen technologies." Journal of Chemical Education 89, no. 8 (2012): 1012-1018.

[8] Savage, Valkyrie, Xiaohan Zhang, and Björn Hartmann. "Midas: fabricating custom capacitive touch sensors to prototype interactive objects." In Proceedings of the 25th annual ACM symposium on User interface software and technology, pp. 579-588. ACM, 2012.

[9] Harris, Amanda, Jochen Rick, Victoria Bonnett, Nicola Yuill, Rowanne Fleck, Paul Marshall, and Yvonne Rogers. "Around the table: Are multiple-touch surfaces better than single-touch for children's collaborative interactions?." In Proceedings of the 9th international conference on Computer supported collaborative learning-Volume 1, pp. 335-344. International Society of the Learning Sciences, 2009.

[10] Rimon, Ori, Haim Perski, Amihai Ben-David, and Rafi Zachut. "Multi-touch and single touch detection." U.S. Patent 8,289,289, issued October 16, 2012.

[11] Cataldi, Pietro, Simeone Dussoni, Luca Ceseracciu, Marco Maggiali, Lorenzo Natale, Giorgio Metta, Athanassia Athanassiou, and Ilker S. Bayer. "Carbon Nanofiber versus Graphene-Based Stretchable Capacitive Touch Sensors for Artificial Electronic Skin." Advanced Science 5, no. 2 (2018): 1700587.

[12] Sell, Johannes K., Herbert Enser, Bernhard Jakoby, Michaela Schatzl-Linder, Bernhard Strauß, and Wolfgang Hilber. "Printed embedded transducers: capacitive touch sensors integrated into the organic coating of metalic substrates." IEEE Sensors Journal 16, no. 19 (2016): 7101-7108.

[13] Abhishek, Kalpattu S., Lee Chun Kai Qubeley, and Derek Ho. "Glove-based hand gesture recognition sign language translator using capacitive touch sensor." In 2016 IEEE International Conference on Electron Devices and Solid-State Circuits (EDSSC), pp. 334-337. IEEE, 2016.

[14] Jiang, Yonggang, Longlong Gong, Xiaohe Hu, Yong Zhao, Huawei Chen, Lin Feng, and Deyuan Zhang. "Aligned P (VDF-TrFE) nanofibers for enhanced piezoelectric directional strain sensing." Polymers 10, no. 4 (2018): 364.

[15] Yang, Ning, Xianping Chen, Tianling Ren, Ping Zhang, and Daoguo Yang. "Carbon nanotube based biosensors." Sensors and Actuators B: Chemical 207 (2015): 690-715. 